\title{
Photomechanic Behavior of Main-chain Type of Polycoumarates
}

\author{
Katsuaki Yasaki, Seiji Tateyama, Daisaku Kaneko, and Tatsuo Kaneko* \\ School of Materials Science, Japan Advanced Institute of Science and Technology, \\ 1-1 Asahidai, Nomi, Ishikawa 932-1292, Japan
}

\begin{abstract}
Hydroxycinnamate derivatives which are polymerizable phenolic acids show two-types photoreactions such as intramolecular $E-Z$ isomerization and intermolecular [2+2] cycloaddition. Although five derivatives of hydroxycinnamic acid were tried to polymerize by a conventional one-bottle method, only two polymers of poly(3-hydroxycinnamic acid) (P3HCA) and poly(3,4-dihydroxycinnamic acid) (PDHCA) were successfully prepared. Because these polymers had cinnamoyl moiety in every main-chain unit, the polymers should show efficient photoreactions by the UV irradiation. The hard-film of these polymers showed a photo-bending behavior. Furthermore, the photo-bending showed generation of high-power $(170 \mu \mathrm{N})$.
\end{abstract}

Keywords: photomechanics, bioplastics, photoreaction, cinnamoyls, phenols

\section{Introduction}

There are a number of biomolecules with optical functions in nature since the light energy is an intrinsic resource for all the living organisms [1-3]. Light can be used as a precise stimulus by adjusting suitable wavelengths, direction and intensity, allowing to be controlled rapidly and remotely. For example, no wires or connections are necessary to use light as a stimulus and then the devices are easily fabricated to reduce their weight. Photo-driven actuators, therefore, are promising in a wide range of micro- and macroscale devices.

One of the photo-driven actuators is composed of a photomechanical polymer. In order to prepare the photomechanical polymeric materials, following requirements are made: 1) to include molecular components effective on twoand three- dimensional actions, 2) well-designed structures to accumulate their forces derived from photochemical reaction to get macroscopic movements. The introduction of an aromatic component into the polymer backbone is an efficient method to intrinsically improve mechanical performance such as Young's modulus, as one can see that aromatic polyesters such as liquid crystalline (LC) polymers have been used in

$\begin{array}{llll}\text { Received } & \text { March } & 27, & 2013 \\ \text { Accepted } & \text { May } & 10, & 2013\end{array}$

the engineering of plastics [4]. If the hard plastic film derived from the polymers with the aromatic backbone show a photomechanical behavior, an efficient motility might be exhibited.

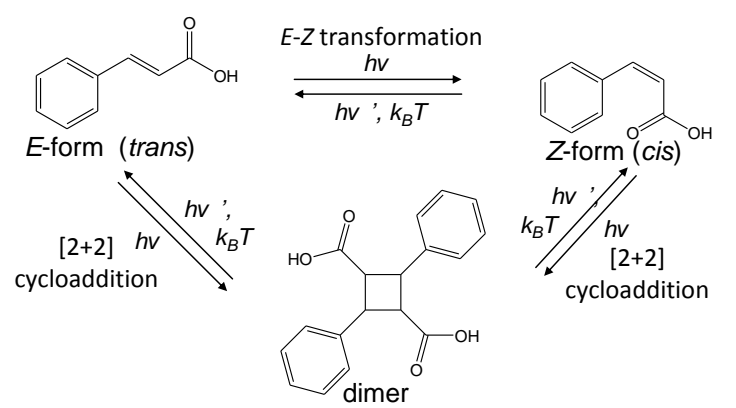

Scheme 1. Photoreaction schemes such as $E-Z$ transformation and $[2+2]$ cycloaddition from both of $E$ and $Z$-forms of cinnamic acid.

Cinnamic acid and its derivatives which were a kind of phenolic compounds exist in plant cell walls as an intermediate metabolite in the biosynthetic pathway of lignin and other biological materials. They show several photoreactions such as [2+2] cycloaddition and $E-Z$ isomerization [5, $6]$. Then cinnamate derivatives are photoreactive and bioavailable components useful to prepare the photomechanical polymers. Here we prepare 
photoreactive polymers as dry and hard materials, where the coumarates are effectively incorporated into their main chains to create aromatic polyesters with rigid backbones.

\section{Experimental}

\subsection{Polymer syntheses}

There are commercially available several coumarates such as 2-hydroxycinnamic acid (2HCA), 3-hydroxycinnamic acid (3HCA), 4-hydroxycinnamic acid (4HCA), 3,4-dihydroxycinnamic acid (DHCA) and 3-methoxy-4-hydroxycinnamic acid (MHCA) (Figure 1). All have high reactivity by carboxyl and hydroxyl groups as well as photoreactive vinylene group, and moreover aromatic ring is incorporated into the corresponding polymer backbones to induce high thermal and mechanical performances. We tried to polymerize these monomers by a conventional one-bottle polycondensation as follows (Scheme 2) [7, 8]. Coumarates as monomer $(14.0 \mathrm{~g}$, about $85 \mathrm{mmol})$ was heated at $50{ }^{\circ} \mathrm{C}$ for $3 \mathrm{hr}$ in the presence of an acetic anhydride $(26 \mathrm{~mL}$ for $2 \mathrm{HCA}, 3 \mathrm{HCA}, 4 \mathrm{HCA}$, MHCA, and $5 \mathrm{ml}$ for DHCA) as a condensation reagent and sodium acetate $(0.14 \mathrm{~g}, 1 \mathrm{w} \%)$ as a catalyst for transesterification. After the monomer was acetylated, the temperature was gradually increased up to $200{ }^{\circ} \mathrm{C}$. The reaction liquid was vacuumed to generate bubbles, presumably due to the elimination of acetic acid condensed as a result of the acidolysis reaction. The molten mixture gradually became viscous upon increasing the reaction period.

\subsection{Confirmation of photoreactivity}

The photoreaction of the coumarate groups was performed by UV irradiation at $\lambda=250-350 \mathrm{~nm}$. The sample which was prepared as solution with DMF was irradiated with a focused UV beam from a Xenon lamp (MAX-303, Asahi Spector Co., Ltd.). The result of photoreactivity was observed by UV-vis spectroscopy.

\subsection{Photomechanical behavior}

P3HCA was dissolved in trifluoroacetic acid (TFA) and N,N-dimethylformamide (DMF) to lead the formation of the cast films from their solutions.

The phototransformations of the polycourmarate films were performed by UV irradiation at $\lambda=$ 250-350 $\mathrm{nm}$. In the photobending observation, the fibers were hung in a horizontal direction by supporter, and then irradiated with a focused UV beam. In another case, the films were hung in vertical direction by supporter and then irradiated with a focused UV beam. The films were irradiated with a focused UV beam from a Xenon lamp. The distance between the fiber and lamp was about 5 $\mathrm{cm}\left(78.0 \mathrm{~mW} / \mathrm{cm}^{2}\right)$. In this operation, the film was cut into rectangles (size: $20 \times 8 \times 0.1 \mathrm{~mm}^{3}$ ).

After revelation of photo-bending of polycoumarate materials, we investigated photoreaction and elucidated the mechanism of photobending. We confirmed photo-reaction of polymer film by IR/ATR methods. IR spectra were took at transformed films both of the UV light irradiated and the non-irradiated faces.

Furthermore, phototransformation ability as photomechanical actuator was investigated as follows. The ability of photoactuator was confirmed by the weight lift up. The glass tube as weight $(17.56 \mathrm{mg})$ was put above P3HCA film (80 $\times 200 \times 0.10 \mathrm{~mm}^{3}$ ), and film was irradiated with a UV beam from over through glass tube weight.

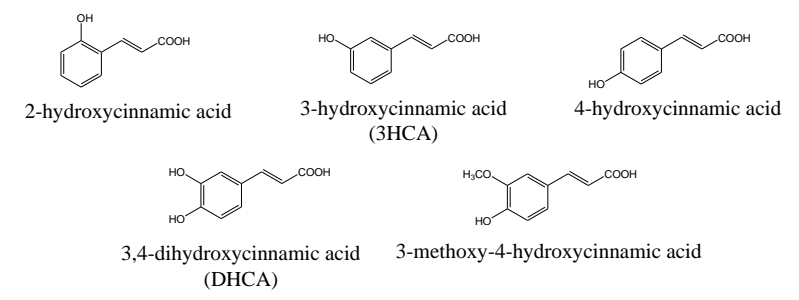

Figure 1. Molecular structures of the hydroxycinnamic acids used as aromatic monomers.

\section{Results and Discussion}

3.1. Confirmation of photoreaction

Photoreaction was confirmed by ${ }^{1} \mathrm{H}$ NMR analyses of a representative cinnamoyl monomer of diacetylated DHCA (Figure 2). The ${ }^{1} \mathrm{H}$ NMR spectrum of the monomer showed a characteristic doublet peak $(J=16.0$ $\mathrm{Hz})$ at a chemical shift of $\delta 6.54 \mathrm{ppm}$, which was assigned to the $\alpha$-proton of vinylene with trans-form. As a result of UV-irradiation, new peaks appeared and one of them a doublet peak $(J=12.8 \mathrm{~Hz})$ at $\delta 6.01 \mathrm{ppm}$ was characteristic. From the coupling constant, this doublet peak was assigned to the $\alpha$-proton of vinylene with a cis-form. On the other hand, a $16 \mathrm{hrs}$ irradiation induced the appearance of NMR signals assigned to cyclobutanes around $4.59 \mathrm{ppm}$. The ratios of isomerization and cycloaddition were calculated from the integral value of each signal and summarized 
in Table 1. As a consequence, it was concluded that $E-Z$ isomerization was faster photoreaction than $[2+2]$ cycloaddition.

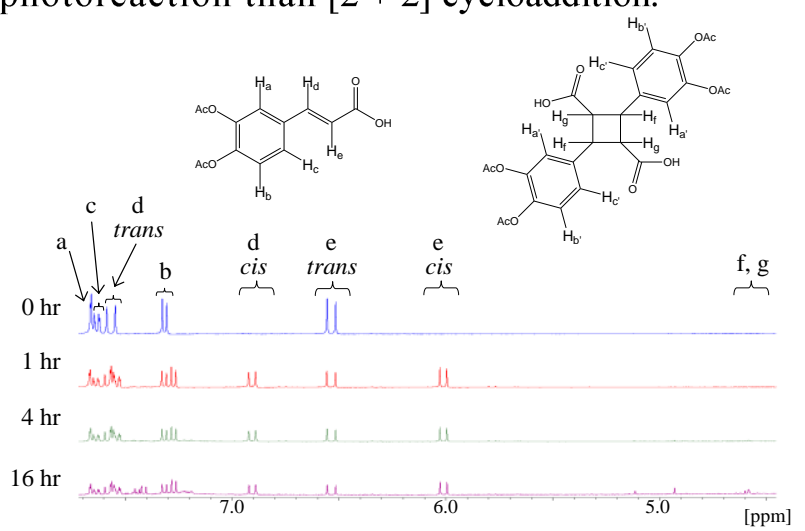

Figure 2. ${ }^{1} \mathrm{H}$ NMR spectra of diacetylated DHCA UV-irradiated for at most $16 \mathrm{hrs}$ measured in DMSO- $d_{6}$.

Table 1. Photoreaction ratio of of AcDHCA by UV irradiation.

\begin{tabular}{ccc}
\hline irradiation time $(\mathrm{hr})$ & $\mathrm{I}$ & $\mathrm{C}$ \\
\hline 0 & 0 & 0 \\
1 & 52 & 0 \\
4 & 48 & 9 \\
16 & 29 & 48 \\
\hline
\end{tabular}

a) $\mathrm{I}$ and $\mathrm{C}$ refers integral ratios; (cis)/\{(trans)+(cis)+ (cyclobutane $)\}$ and (cyclobutane $) /\{($ trans $)+($ cis $)+$ (cyclobutane) $\}$, respectively.

\subsection{Polymer syntheses}

We tried to polymerize coumarate monomers 4HCA, MHCA, 2HCA, 3HCA, and DHCA by a conventional one-bottle method. The polymerization of two monomers 4HCA and MHCA with 4-substituted $\mathrm{OH}$ groups did not proceed well to get only oligomers, presumably due to too rigid chains showing no melting or solubility in any solvents. On the other hand, 2HCA which has 2-substituted $\mathrm{OH}$ group did not proceed well to get only oligomers, presumably due to too strong steric hindrance. However, the $3 \mathrm{HCA}$ was proceeded at some extent to create poly(3-hydroxycinnamic acid) (P3HCA). P3HCA was dissolved in TFA and other solvents. Furthermore, polymerization of DHCA which has $\mathrm{AB}_{2}$ style polyfunctionality proceeded at some extent to create poly(3,4-dihydroxycinnamic acid) (PDHCA). In general, $\mathrm{AB}_{2}$ style polyfunctional monomer was known to become hyper branched polymer [9]. Solubility of PDHCA was different from that of P3HCA. For example, the solubility of PDHCA was not so good, but it dissolved in DMF and TFA. The poor solubility might be attributed to too rigid $p$-connected polyarylate structures.

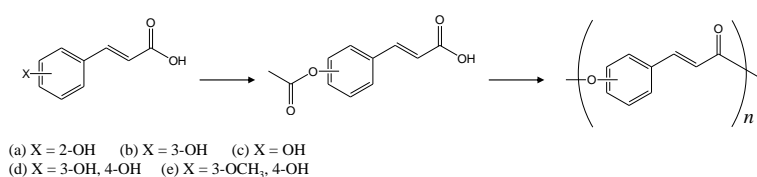

Scheme 2. Synthesis of acetylated coumarates and their polymerization.

The achievement of polymerization by acidolysis reaction was confirmed by FT-IR. In Figure 3, FT-IR spectra of Ac3HCA and AcDHCA showed the peaks at 1674-1678 and $1755-1756 \mathrm{~cm}^{-1}$ which were assigned to carboxylic acids and saturated esters, respectively. On the other hand, P3HCA and PDHCA spectra showed the peaks at $1719-1731 \mathrm{~cm}^{-1}$ which were assigned to unsaturated esters. These results confirmed acidolysis reaction by the disappearance of carboxylic acid and appearance of unsaturated ester. Moreover unsaturated esters of PDHCA were duplicated, which suggested the formation of $p$ and $m$-substituted esters.

The molecular weight of P3HCA and PDHCA were estimated by gel permeation chromatography (GPC, polystyrene standard) in DMF. The number-average molecular weights $\left(M_{\mathrm{n}}\right)$, weight-average molecular weights $\left(M_{\mathrm{w}}\right)$ and molecular weight dispersion $\left(M_{\mathrm{n}} / M_{\mathrm{n}}\right)$ of P3HCA were determined as $3.04 \mathrm{x}$ $10^{4}, 5.10 \times 10^{4}$ and 1.68 , respectively. $M_{\mathrm{n}}, M_{\mathrm{w}}$ and $M_{\mathrm{n}} / M_{\mathrm{n}}$ of PDHCA were determined as $2.87 \times 10^{4}, 4.88 \times 10^{4}$ and 1.70 , respectively. Both of them have molecular weight high enough to process into films.

\subsection{Confirmation of photoreactivity}

The photoreactivity of P3HCA and PDHCA were investigated by measuring the UV-vis absorption spectra of the UV-irradiated specimens, and the results are summarized in Figure 4a. The peak appeared at $\lambda_{\max }=288 \mathrm{~nm}$ in the UV-vis spectrum of the P3HCA solution and at $\lambda_{\max }=300$ $\mathrm{nm}$ in that of the PDHCA solution before UV irradiation. From the polymer structure, it was concluded that these absorbances were assigned to $\pi-\pi$ transition of conjugated cinnamate electrons. The peak intensities were reduced by the irradiation as shown in the left spectra of Figure $4 \mathrm{a}$ and $b$. The absorbance of the cinnamate groups almost equilibrated at $40 \mathrm{~min}$. Many researchers have reported the similar phenomena in UV absorbance of the cinnamate group with UV irradiation time due to formation of cyclobutane 
a

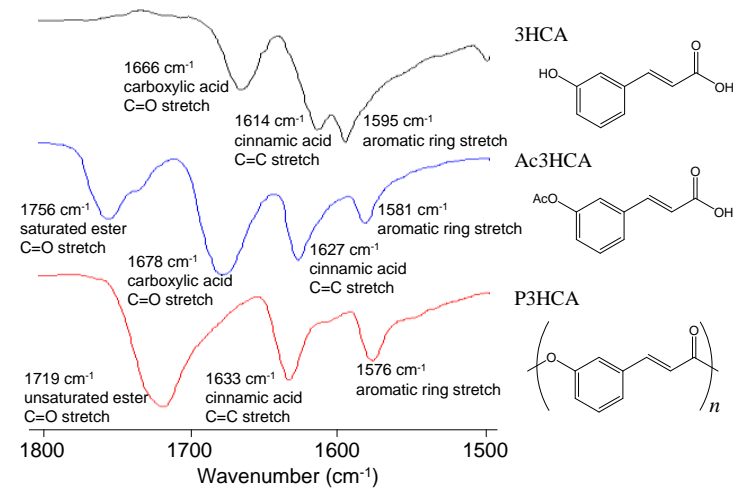

b

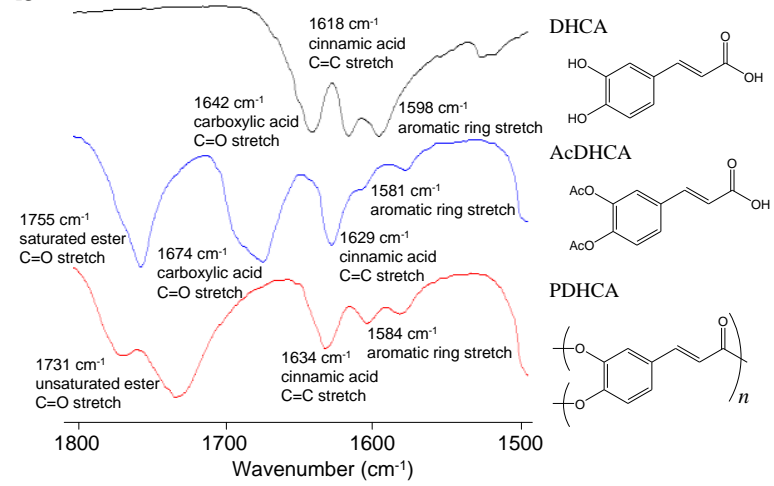

Figure 3. IR spectra of cinnamate monomers and polymers. (a) $3 \mathrm{HCA}$, acetylated $3 \mathrm{HCA}$, and P3HCA. (b) DHCA, acetylated DHCA, and PDHCA.

a

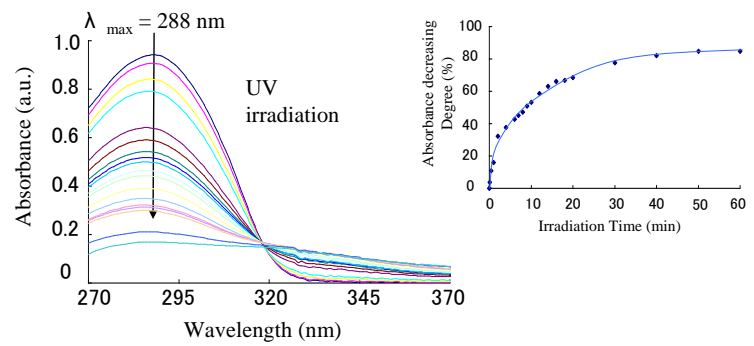

b

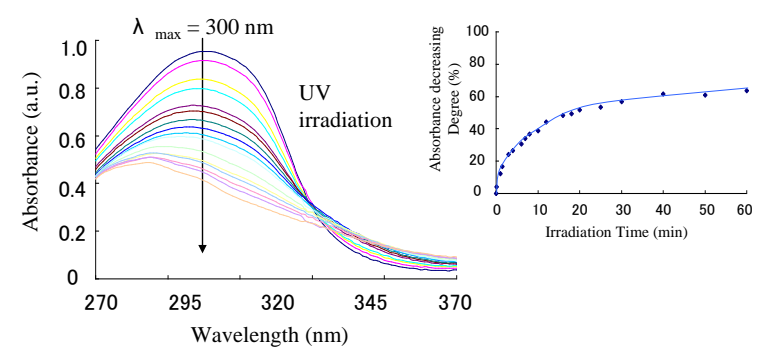

Figure 4. Changes in UV-vis spectra (left) and crosslinking degrees (right) of (a) P3HCA and (b) PDHCA, measured in DMF as a function of UV irradiation time. Cross-linking degree was determined from the ratio of UV-vis absorbance value of UV-irradiated samples to non-irradiated one. bonds [10-12]. Absorbance changes at wavelength of $\lambda_{\max }$ (P3HCA: $\lambda_{\max }=288 \mathrm{~nm}$, PDHCA: $\lambda_{\max }=$ $300 \mathrm{~nm}$ ) were converted into cross-linking degree and plotted against irradiation time (right of Figure 4). The cross-linking degree was saturated after 40 min of irradiation, and eventually about $80 \%$ of the cinnamate groups in the solution were cross-linked by UV-irradiation.

\subsection{Photomechanical behavior}

We tried to observe the photodeformation of the P3HCA materials using the cast films. The film was cut into rectangles (size: $20 \times 8 \times 0.1 \mathrm{~mm}^{3}$ ).
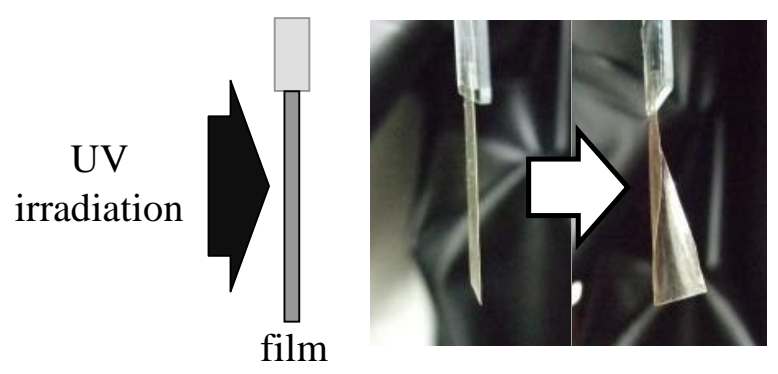

Figure 5. Photomechanical behavior of P3HCA films. Film was hung by supporter and irradiated from left.

Young's modulus of the film was $1.8 \mathrm{GPa}$ and much higher than elastomers. The edge of the film was attached at a supporter and hung as shown in Figure 5. The Xenon lamp was then shone onto the film surface. We found that the film gradually bent in a direction away from the lamp. Here we confirmed no temperature gradient and no solvent vaporization from the film. If the Xenon lamp was turned off, the bending motion stopped immediately.

Figure 6 shows the IR-ATR spectra of the surface for P3HCA films UV-irradiated for $0,1,10$ and $100 \mathrm{~min}$. The distinct IR peaks assigned to vinylene bonds [-HC $=\mathrm{CH}-]$ appeared at 1650,1430 , and $980 \mathrm{~cm}^{-1}$ in the spectra of the non-irradiated film. After $1 \mathrm{~min}$ of irradiation, the spectrum seemed to show no intrinsic change as compared with the spectrum of the non-irradiated film. On the other hand, the absorption peak at $1430 \mathrm{~cm}^{-1}$ shifted slightly to a higher wavenumber and became weak. Furthermore, all of the peaks became broadened after $10 \mathrm{~min}$ of irradiation. With longer irradiation times (100 min), the IR peaks became broader. These peak changes indicate that the vinylene peaks reacted, and the P3HCA structure was disordered, presumably because structural variation by E-Z isomerization and $[2+2]$ cycloaddition. The previous study on photoreaction 
of P4HCA showed the polymer was photo-cross-linked very efficiently because the liquid crystalline alignment enhanced the interchain cycloaddition reaction $[7,13]$. On the other hand, P3HCA exhibited no liquid crystalline phase but formed amorphous structure. In order to form the cyclobutane ring by the dimerization of vinylene group of cinnamoyls, the structural intermolecular matching of vinylene $\pi$-electrons were indispensable. In P3HCA, it was considered that such a matching may be very difficult in the amorphous structure. As a consequence, E-Z isomerization occurred predominantly to induce the film bending with facing its convex face toward the Hg-lamp.

Furthermore, we tried to make a test of load-lift. As illustrated in the left of Figure 7, glass tube with a weight of $17 \mathrm{mg}$ was put over the P3HCA

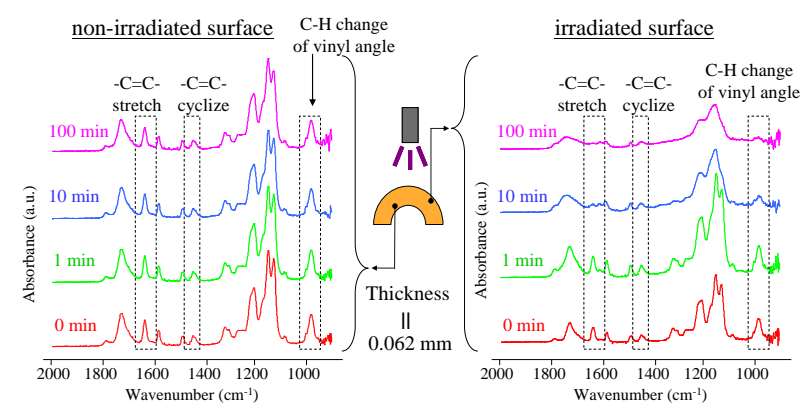

Figure 6. IR/ATR spectra of UV-irradiated face (right) and opposite one (left) of P3HCA film.

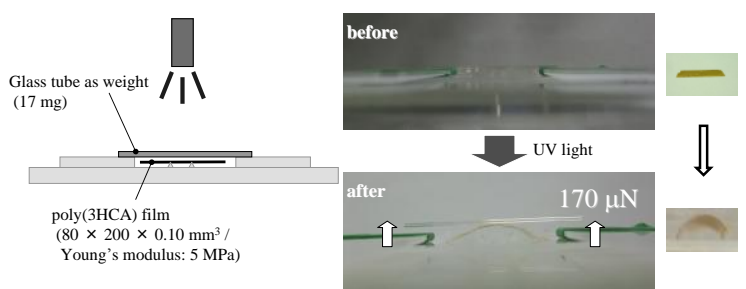

Figure 7. Photomechanical behavior of P3HCA films. Left is an illustration for lift-up test; glass tube was put over the film as a load and UV-irradiated from above. Right pictures were taken before and after lift-up. The small pictures at the right: film deformation by UV-irradiation.

film, and then high-pressure Hg-lamp was irradiated. As a result, the film lifted up the load of glass tube accompanied with a mechanical force about $170 \mu \mathrm{N}$, which was a $\mu \mathrm{N}$-scale photomechanical work of the hard plastic film.

\section{Conclusion}

A series of the polycoumarates which had formability and photo reactivity were developed, and their films showed photomechanic behavior; gradual bending by UV-irradiation. These photo transformations were explained by effective photo reaction of vinylene group derived from cinnamoyl group in polymer main-chain. This finding will lead to a direct energy conversion from light to mechanical works.

\section{References}

1. K. C. Yeh, S. H. Wu, J. T. Murphy, J. C. Lagarias, Science, 277 (1997), 1505-1508.

2. P. E. Kendrick, G. H. M. Kronenberg, Photomorphogenesis in Plants, Springer, (1994).

3. M. Furuya, Annu. Rev. Plant Physiol. Plant Mol. Biol., 44 (1993), 617-645.

4. Y. Imai, High Perform. Polym., 7 (1995), 337-345.

5. D. Hernanz, V. Nunez, A. Asncho, C. Faulds, G. Williamson, B. Bartolome, C. Gomez-Cordoves, J. Agric. Food Chem., 49 (2001), 4884-4888.

6. W. D. Hoff, P. Dux, K. Hard, B. Devreese, I. M. Nugteren-Roodzant, W. Crielaard, R. Boelens, R. Keptein, J. Beeumen, K. J. Hellingwerf, Biochemistry, 33, (1994), 13959-13962.

7. T. Kaneko, M. Matsusaki, H. T. Tran, M. Akashi, Macromol. Rapid. Commun., 25 (2004), 673-677.

8. T. Kaneko, Chem. Rec., 7 (2007), 210-219.

9. G. Yang, M. Jikei, M. Kakimoto, Macromolecules, 32 (1999), 2215-2220.

10. D. Kaneko, K. Matsumoto, T. Kaneko, Plant Biotechnology, 27 (2010), 293-296.

11. D. Kaneko, S. Wang, K. Matsumoto, S. Kinugawa, K. Yasaki, D. H. Chi, T. Kaneko, Polym. J., 43 (2011), 855-858.

12. D. Kaneko, K. Matsumoto, S. Kinugawa, S. Tateyama, T. Kaneko, Polym. J., 43 (2011), 944-947.

13. T. Kaneko, H. T. Tran, D. J. Shi, M. Akashi, Nature Material. 5 (2006), 966-970. 